EUROPEAN COOPERATION IN

THE FIELD OF SCIENTIFIC

AND TECHNICAL RESEARCH
COST 252 TD (98) 38

Venice, Italy, 6 November 1998

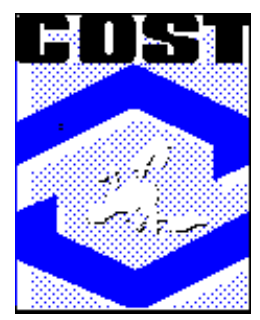

EURO - COST

SOURCE: $\quad$ SRU (University of Surrey)

TASK: $\quad$ WG2

KEYWORDS: $\quad$ MULTIMEDIA, S-ATM, CALL PERFORMANCE

\title{
SATELLITE-ATM NETWORKING AND CALL PERFORMANCE EVALUATION FOR MULTIMEDIA BROADBAND SERVICES
}

\author{
I. Mertzanis, G. Sfikas, R. Tafazolli, B. G. Evans
}

Centre for Communication Systems Research (CCSR), University of Surrey, Guildford, Surrey, GU2 $5 X H, U . K$.

Tel: ++44(0)1483-259844, Fax:++44(0)1483-259504, Email: \{I.Mertzanis,

G.Sfikas\}@ee.surrey.ac.uk

\begin{abstract}
In this paper, we identify the protocol reference model that best satisfies the requirements of an integrated solution of the future broadband satellite networks with the Broadband-Integrated Services Digital Network (B-ISDN). In addition, the Connection Admission Control (CAC) mechanism that needs to be implemented to satisfy certain Grade Of Servicel (GoS) and Quality Of Service ${ }^{2}(Q o S)$ criteria is discussed. A new methodology for the call performance evaluation of the Available Bit Rate (ABR) service class in a satellite Asynchronous Transfer Mode (ATM) network is proposed. Results at call level for different mix of service classes are presented using both analytical and simulation models. The simulation model was developed using OPNET modeller and it was used to record statistics that cannot be easily obtained by analysis.
\end{abstract}

\footnotetext{
${ }^{1}$ Defined as the call blocking probability

${ }^{2}$ Defined in terms of Cell Loss Rate (CLR) and Cell Delay Variation (CDV)
} 


\section{INTRODUCTION}

Satellites are foreseen as being complementary to the future fixed or terrestrial mobile networks in order to provide coverage to remote users. On the one hand, current work in the European ACTS (Advanced Communications Technologies and Services) [1] projects such as SECOMS ${ }^{3}$, ASSET ${ }^{4}$,

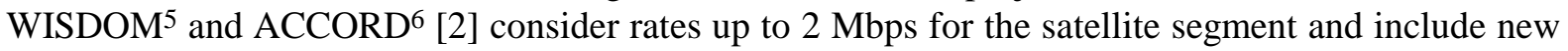
generation multi-spot beam satellites with advanced on-board processing capabilities. On the other hand, targeting at high data rate multimedia services mainly to fixed and possibly portable user terminals a large number of Ka-band $(30 / 20 \mathrm{GHz})$ proposals exist such as: SPACEWAY, ASTROLINK [3], CYBERSTAR [4], TELEDESIC [5], N-STAR [6], WEST [7] and EUROSKYWAY [8]. As suggested in [9], a new modified version of the ATM protocol layer (known as Satellite-ATM layer, S-ATM) is required. This proposal has been further exploited and a highly integrated broadband Satellite-ATM network scenario is presented.

The rest of the paper is organised as follows: In the following two sections the satellite network architecture and the protocol stacks are discussed. Section four presents the resource management block diagram and the mapping of the ATM layer services to the Medium Access Control (MAC) layer ones. The methodology that has been developed is explained in section five, along with the assumptions made. The paper concludes with the calculation of the ATM and MAC layer resources as functions of the offered load, for two different cases.

\section{Broadband SATEllite Network ArChitecture}

The satellite constellation is the initial parameter that affects the design of a global network infrastructure. Most of the proposed systems, concentrate on the Geostationary Orbit (GEO). For example: in Europe EUROSKYWAY by Alenia Aerospazio (Italy) and WEST (GEO component) by Matra Marconi Space (France/UK), in the United States ASTROLINK by Lockheed Martin, SPACEWAY by Hughes and CYBERSTAR (GEO component) by Loral Space and in Japan N-STAR by NTT. The non-GEO systems are: TELEDESIC that proposed to use 288 Low Earth Orbit satellites (LEO) and CELESTRI (LEO 63) which was recently merged with the TELEDESIC proposal. At the same time, there are proposals based on hybrid constellation approach using GEO and non-GEO components; e.g. WEST and CYBERSTAR. As far as the satellite ground network is concerned, the number and the location of the Land Earth Stations (LES) depends on the constellation parameters and on the provision of Inter-Satellite Links (ISLs) as well as the feeder link bandwidth requirements. Another parameter that influences the selection of the satellite network architecture, is the dependency on the terrestrial network infrastructure. The satellite links, are essential for inter-station signalling when there is no terrestrial infrastructure deployed. For example, most GEO systems do not need ground station interconnection through terrestrial links, whereas non-GEO systems require only a few satellite links to the LESs when ISLs are used, otherwise they are highly dependent on a fast backbone network. In GEO systems, ISLs are considered mainly to handle the traffic between different regions of the earth and to bypass the terrestrial links whereas in most non-GEO constellations ISLs are essential in order to reduce the number of LESs. In a typical broadband satellite system (see Figure 1) the following network entities are considered:

- User Terminals (UT): Several different protocol standards such as: ATM User Network Interface (ATM-UNI), Frame Relay UNI (FR-UNI), Narrowband Integrated Services Digital Network (NISDN) Basic Rate Interface (BRI), N-ISDN Primary Rate Interfaces (PRI), Transmission Control

\footnotetext{
${ }^{3}$ Satellite EHF Communications for Mobile Multimedia Services

${ }^{4}$ ACTS Satellite Switching End-to-end Trials

${ }^{5}$ Wideband Satellite Demonstration Of Multimedia

${ }^{6}$ ACTS Broadband Communication Joint Trials and Demonstration
} 
Protocol / Internet Protocol (TCP/IP). They are connected to the Satellite Adaptation Unit (SAU) through one of the supported standard interfaces.

- Satellite Adaptation Unit (SAU): This is a non-standard, specially designed unit, responsible to provide access to the satellite network. It performs all the necessary user terminal protocol adaptations to the satellite protocol platform. The SAU also includes all the physical layer functions such as channel coding, modulation/demodulation, the Radio Frequency (RF) parts and the antenna section. A set of different types of terminal with a variety of transmission capabilities

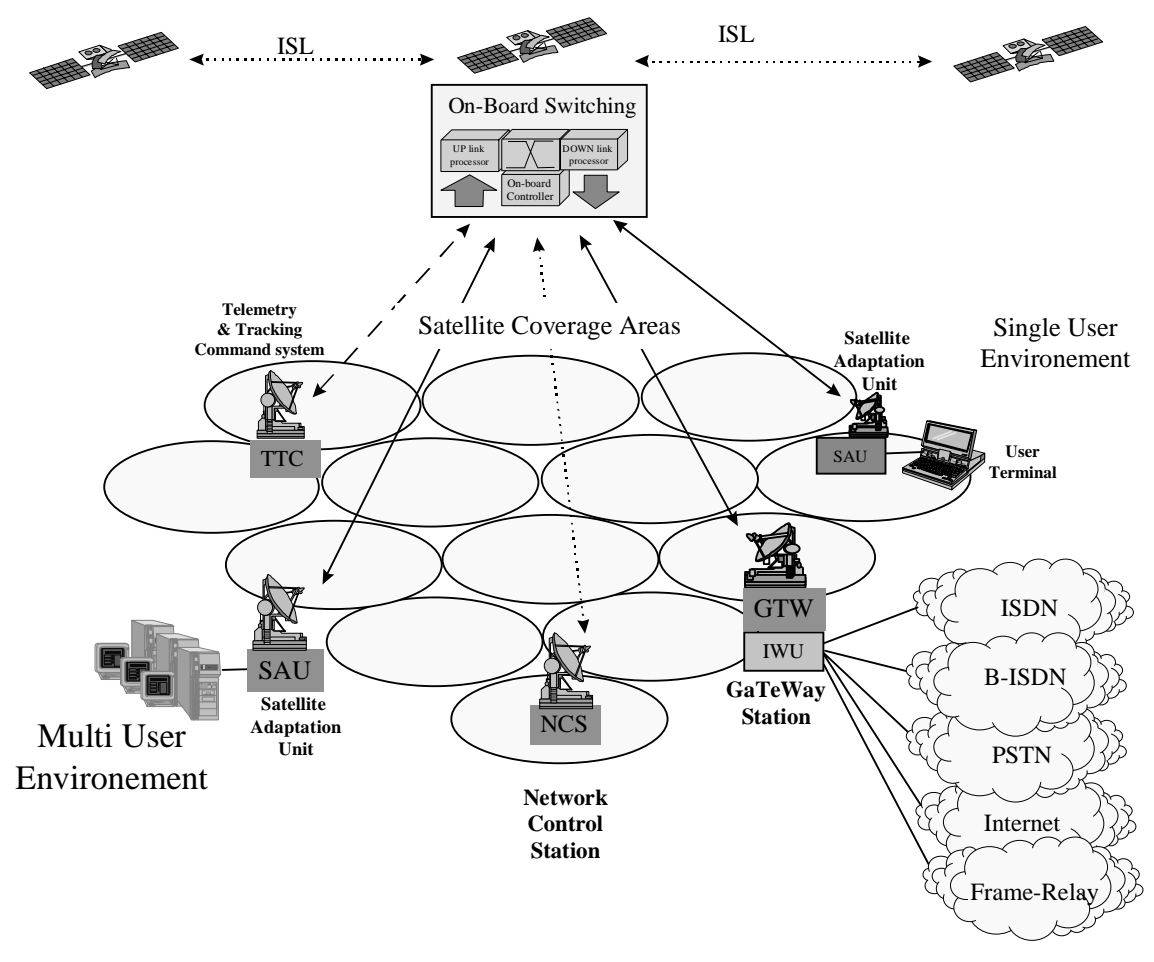

Figure 1: Satellite network architecture and connectivity with the fixed networks.

is usually offered by a satellite network. Starting from minimum transmission rates of $16 \mathrm{Kbps}$ of even less, they can cope with maximum transmission rates of $144 \mathrm{Kbps}$ or $384 \mathrm{Kbps}$ for personal type user terminals, or even $2,048 \mathrm{Kbps}$ and higher for fixed type terminals with larger antennas. All of the supported terminals, share the same the access scheme and protocols stacks.

- Payload $(P / L)$ : Full on-board satellite signal re-generation is assumed in most of the future broadband satellite systems. The on-board satellite processing units perform multiplexing, demultiplexing, channel coding/decoding and ATM 'like' switching using a multi-spot beam configuration. The on-board satellite switch includes only part of the functions that a ground ATM switch would perform. Most of the power hungry processing operations such as the call set-up signalling or the Connection Admission Control are performed on the ground.

- Gateway stations (GTW): These are the Land Earth Stations (LES) that provide connectivity to the external networks. Most of the future proposed broadband satellite systems support interconnection to the fixed B-ISDN, Frame Relay, N-ISDN, PSTN and Internet, via the proper interworking units at the gateway. The level of interworking between the GTWs and the terrestrial networks depends on the type of traffic that the satellite network carries. The SAU provides an access interface very similar to the standard ATM-UNI, therefore the required interworking at the terminal or at the GTW sides is minimised. The signalling protocols for call and connection control can be reused (based on the ITU-T Q.2931 standard) and the traffic and network management functions can share common characteristics. All ATM service classes can be supported and directly mapped into the satellite air interface through the SAU. Finally, the 
interconnection interfaces with all the other public terrestrial networks should be based on standards for interworking with B-ISDN. In GEO systems the placement and number of GTWs on the ground segment depend mainly on the traffic demand. A large number of gateways is expected in geographical areas where the traffic demand is high and these gateways are always connected with the same satellite(s). However, in non-GEO systems the number and placement of the gateway stations depends on some additional system design characteristics such as: constellation design, use (or not) of ISLs and the overall end-to-end system delay budgets. For example, in a global MEO system with no inter-satellite links, a total number of less than 10 gateways can provide full connectivity to the land masses most of the time. A LEO system will require tens to hundreds of gateways but this number can be reduced with the use of ISLs.

- Network Control Station (NCS): A central entity, used in a GEO satellite system (usually one per satellite) that provides overall control of the satellite network resources and operations. This node is responsible for allocating radio resources to the LESs/GTWs according to a long term resource planning scheme. The NCS is responsible for performing most of the resource management, call management and user/terminal mobility management functions in addition to authentication, registration, de-registration and billing. In some systems, these operations are performed in more than one ground stations in a distributed manner.

\section{Satellite Access Interface and Protocol Stacks}

The proposed protocol stacks for both the control and the user plane in a satellite ATM network are shown in Figure 2. In [9], the use of the S-ATM layer has been proposed for the space segment. The Medium Access Control and the radio physical layers reside below the S-ATM. The fields that are present in the S-ATM cell header carry essential routing and control information for the satellite segment and depend on the implementation of the on-board satellite switch. The dimensioning of the Satellite Virtual Path Identifier (S-VPI) and the Satellite Virtual Channel Identifier (S-VCI) fields

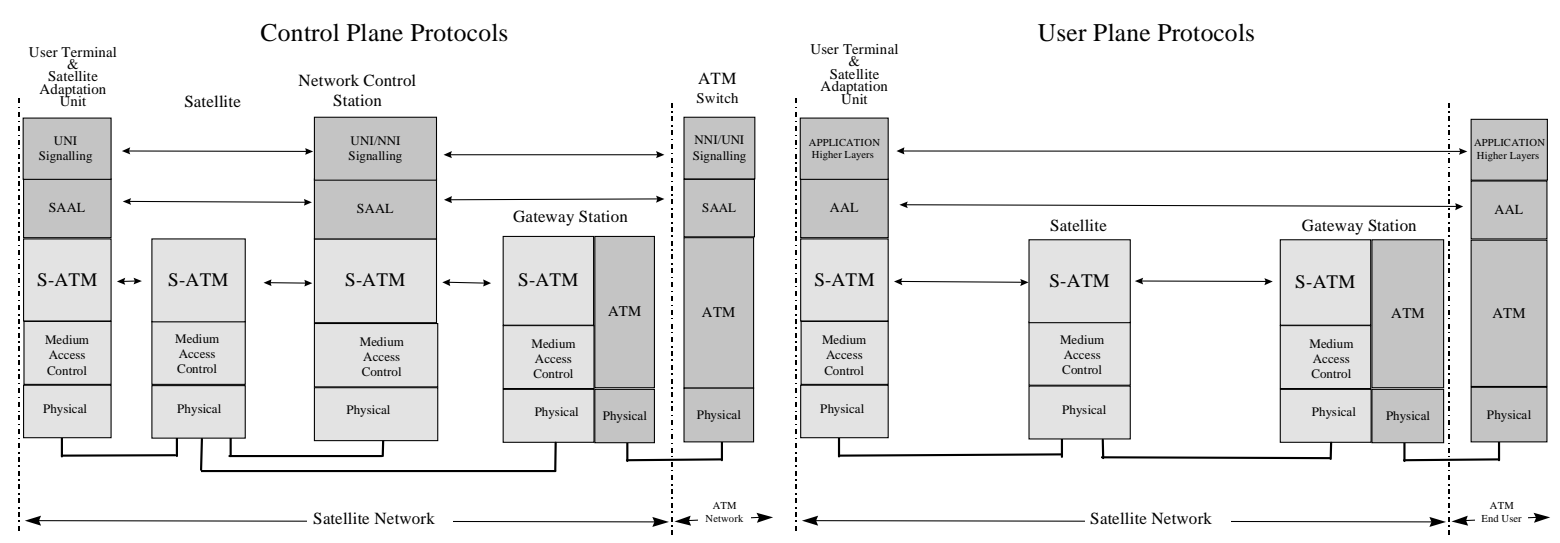

Figure 2: ATM based protocol reference model

depends on system parameters such as: the satellite capacity, the number of spot-beams, the satellite terminal transmission granularity and the switch architecture.

Signalling for call control is based on the Q.2931 protocol standard and is terminated on the ground segment. In the case of mobile or portable terminals, future standards by ATM Forum or ITU-T for B-ISDN signalling and Intelligent Network (IN) can be supported in a highly integrated network environment. All the satellite specific functions can be defined inside the S-ATM layer and control messages can be transmitted through the F4 and F5 Operation and Maintenance (OAM) cell flows. 


\section{Resource Management and Control}

One of the main areas of interest in broadband teletraffic engineering is the provision of multimedia services with QoS guarantees to a large number of users while maintaining high system resource utilisation. In systems where user applications can share common network resources some level of connection admission control is required in order to provide fairness and to guarantee that the requested QoS criteria are met. In a broadband satellite network a two level call acceptance control algorithm must be considered; the first at the MAC layer and the second at the ATM layer. As shown in Figure 3, the resource management and control functions are distributed between the space and the ground segment. The on-board satellite switch is responsible to provide full connectivity from any up-link to any down-link spot beam and it is controlled by the switch control unit and the Call Control (CC) and the CAC units. The physical location of the blocks that implement the CC and the $\mathrm{CAC}$ functions can be on the ground in order to reduce the on-board processing requirements. The up-link and down-link resource control units are responsible for the incoming and outgoing traffic management actions at the input and the output ports of the ATM switch respectively. The overall network resource management function is performed by the Resource Management (RM) controller which supervises the operation of the MAC and the ATM resource managers.

\subsection{Mapping ATM service classes into the air interface}

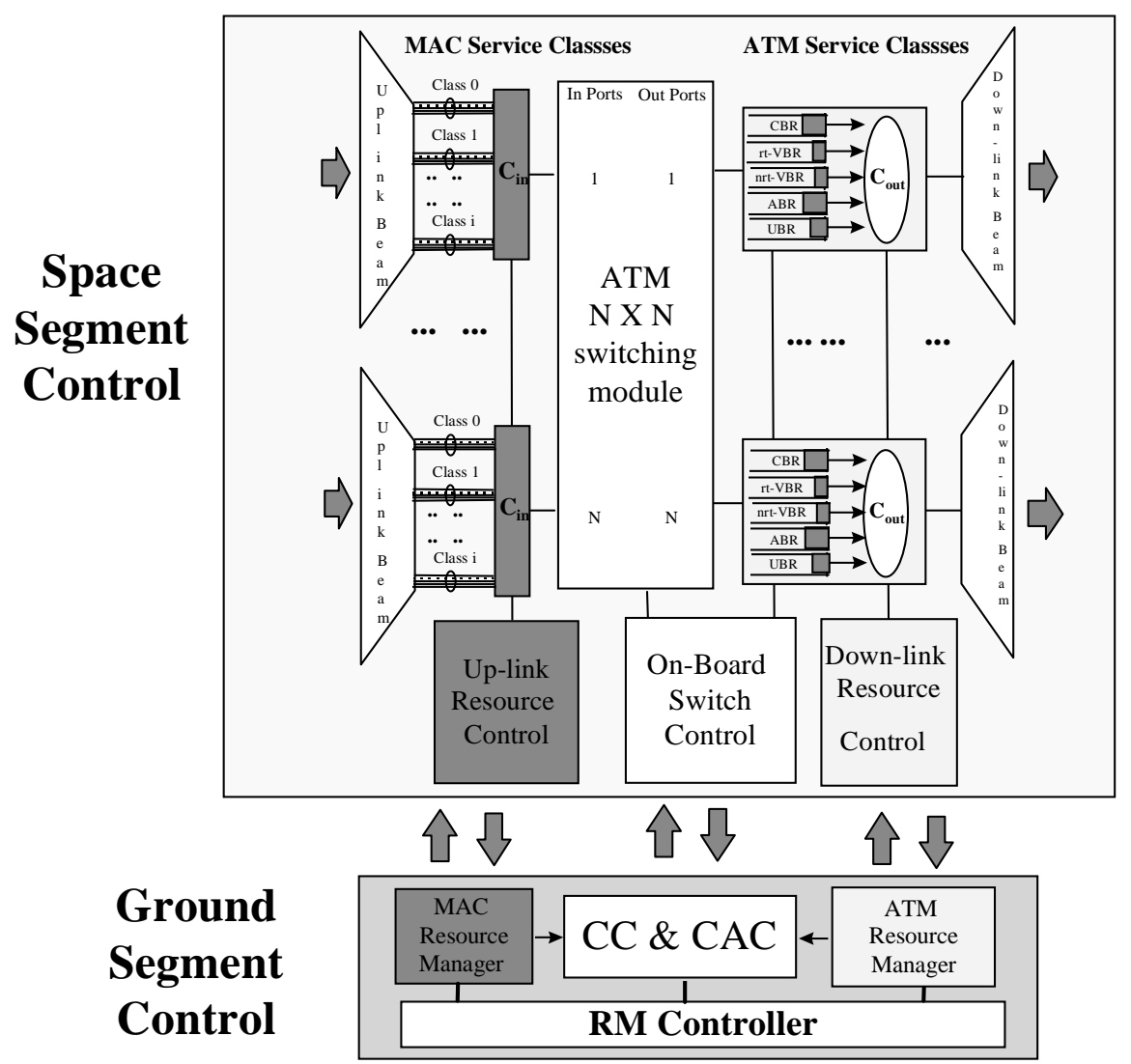

Figure 3: Resource Management and Control block diagrams

The flexibility in designing a MAC scheme for a GEO satellite system, is limited mainly by the long propagation delay. Therefore, in most of the proposed schemes at least a minimum up-link bandwidth reservation takes place for most of the supported service classes. All services can be classified at the MAC layer according to the number of slots that are assigned in the up-link direction in addition to the time period for which the resources are allocated (i.e. permanent, semi-permanent, on demand). For bi-directional services each link is set-up independent from each other but in our study we did not 
take the forward traffic requirements into consideration. A Multi Frequency Time Division Multiple Access (MF-TDMA) access scheme is considered in our study. Five distinct service categories have been specified by the ATM Forum [13] to accommodate all the different applications: Constant Bit Rate (CBR), rt-VBR (real-time Variable Bit Rate), nrt-VBR (non-real time VBR), Unspecified Bit Rate (UBR) and Available Bit Rate (ABR). We assume that all service classes except the UBR share the same pool of up-link resources. This is because UBR is the only service that a hybrid approach which combines both random access and a reservation based resource allocation algorithm should be used, if we wish to provide a certain level of QoS.

During the call set-up phase, the CBR, rt-VBR and nrt-VBR services are assigned a fixed number of up-link slots which remain constant for the duration of the call. Therefore, some multiplexing gain can be achieved only at the ATM layer by limiting the available resources at the ATM switch output queues. For the ABR service, a more flexible access scheme is proposed. An ABR call is accepted or blocked at the MAC layer according to its Initial Cell Rate (ICR), or Minimum Cell Rate (MCR) requirements. After the call is accepted, all the slots that remain free in the up-link direction can be shared among all ABR users in order to satisfy the instantaneous requirements for increased bandwidth. If we want to maintain certain call blocking rates for all the supported service classes at the MAC layer, the available slots which are shared among all ABR services should be used in such away, that does not affect the new call blocking rate of any service type.

The determination of the call blocking probabilities of $\mathbf{N}$ different service classes each one requesting for constant rate of $c_{i}$ from a shared link of capacity $\mathbf{C}$ can be performed with some widely used multi-rate models if a complete sharing admission policy is assumed. Each service class is characterised by an arrival process which is assumed to be Poisson with mean call arrival rate $\lambda_{\mathbf{i}}$ and call holding times following a general distribution with mean call holding times $\mu_{\mathrm{i}}$. The analysis of multi-rate models is well presented in existing literature and product form solutions for the state probabilities can be found in $[10,11]$. However, the solution to this problem becomes intractable as the number of states and service classes increases. Therefore, a recursive solution that was given to this problem by [12] simplifies the calculations for a large number of service classes and system states. Both the unnormalised probability $\mathbf{P}_{\mathbf{u n}}(\mathbf{m})$ of $\mathbf{m}$ occupied bandwidth units and the normalised probability $\mathbf{P}(\mathbf{m})$ are given by:

$$
P_{u n}(m)=\left\{\begin{array}{lrl}
1 & : \mathrm{m}=0 \\
0 & : \mathrm{m}<0 \\
\frac{1}{m} \sum_{i=1}^{N} P_{u n}\left(m-m_{i}\right) \cdot m_{i} \cdot \frac{\lambda_{i}}{\mu_{i}}: 0 & <\mathrm{m} \leq \mathrm{M}
\end{array} \quad P(m)=P_{u n}(m)\left(\sum_{m=0}^{M} P_{u n}(m)\right)^{-1}\right.
$$

where $\mathrm{M}=\mathrm{C} / \mathrm{dc}, \mathrm{m}_{\mathrm{i}}=\mathrm{c}_{\mathrm{i}} / \mathrm{dc}$ and $\mathrm{dc}$ represents the greatest common divisor of $\mathrm{c}_{\mathrm{i} .}$. The blocking probability per class can be calculated from:

$$
P_{b i}=\sum_{m=M-m_{i}+1}^{M} P(m)
$$

\section{Call Performance evaluation}

Each connection in an ATM network is associated with a set of traffic parameters in addition to a set of negotiated and non-negotiated QoS parameters [13]. During the call establishment phase, one important network operation is the evaluation of the QoS impact of the new call on the accepted calls that share common resources. CAC is the network function that decides if a new call should be accepted or not. A new call can be blocked if there are not enough resources either at the MAC or at the ATM layer. Analytical models can be very complex when considering multi-rate services at both call and cell level and a simulation model that runs at cell level will require extremely long processing period to produce accurate results at call level. Therefore, a hybrid approach was taken 
that combines a call level simulation of multi-rate, multi-class services and incorporates look-up tables that provide the ATM resource utilisation statistics for different ATM service classes. These tables can be generated by using proper analytical or simulated traffic source modelling techniques depending on the service type.

\subsection{CBR Service}

A fixed number of up-link slots are allocated for a CBR call; this number corresponds to the source Peak Cell Rate (PCR). No multiplexing gain can be performed at the MAC layer from terminals that share the same up-link resources due to the real time constraints. However, depending on the activity of the source, multiplexing gain can be achieved at the ATM layer. For the simulation it was assumed that voice traffic was supported over the CBR service. Each source was modelled as an ON-OFF process with negative exponential call holding time and then the analysis presented in [14] was used to obtain the utilisation of the allocated resources as a function of the number of users, for different QoS (expressed in terms of CLR and maximum queuing delay, at the ATM switch).

\section{2 nrt-VBR}

The nrt-VBR service is intended for non real time applications that have bursty traffic characteristics and characterised in terms of PCR, Sustainable Cell Rate (SCR) and Maximum Burst Size (MBS). For the ATM cells that are transferred within the traffic contract, the application expects low CLR. To simplify our calculations we assume that the MAC resources are allocated in a similar way to the CBR service (circuit switch allocation using the SCR instead of the PCR). No multiplexing gain can be obtained at the ATM level, since detailed traffic sources for this service have not been implemented. The SCR is also used at the ATM layer to allocate (ATM) resources to each user. The user is responsible for managing its own transmission rate depending on the terminal buffer size.

\section{3 rt-VBR}

Real-time VBR services are also treated similar to the CBR service at the MAC layer. However, the number of the allocated up-link slots is related to the SCR and not to the PCR. For the simulation it was assumed that video traffic was supported over the rt-VBR service. The traffic was modelled as in [15]. Similar to CBR, the resource utilisation was calculated as a function of the number of users and the expected QoS, in order to consider the multiplexing gain at the switch.

\subsection{ABR Service}

During the connection set-up phase, the following traffic parameters are negotiated for the $A B R$ service types: the Initial Cell Rate (ICR), the Minimum Cell Rate (MCR) and the Peak Cell Rate (PCR). An ABR source is allowed to transmit cells at an Allowed Cell Rate (ACR), which varies over time depending on the network congestion status. The mechanism to change the source ACR is based on the transmission of RM cells that traverse end-to-end from the source to destination (forward direction) and are looped back to the originating source in the backward direction. The contents of the RM cells can be changed by the destination or the intermediate nodes by altering the Congestion Indication (CI) bit and the Explicit Rate (ER) fields and the source ACR is adjusted according to these fields. The network congestion indication mechanism depends on the switch architecture and can be Explicit Forward Congestion Indication (EFCI)-based switch or ER-based switch. A set of time-out mechanism exists in this type of flow control to overcome the problems arising from erroneous cells. The efficient operation of a rate-based congestion control mechanism depends on the careful switch buffer dimensioning procedure in order to avoid cell loss. In general, ER based switches perform better than the EFCI but are harder to be implemented. For long end-toend control loops such as those that are included in a GEO satellite network, it is suggested that smaller control segments should be considered (at least two segments must be created; one for the radio part and one for the fixed part of a connection. In this paper, only the radio parts of all ATM connections were considered and no particular algorithm (either at the MAC or at the ATM layer) that supports bandwidth on demand for ABR services has been taken into account. Therefore, the 
simulation model was used mainly to produce statistics that express the resources that remain free to be used by the ABR services at both the MAC and the ATM layers as a function of the total offered load under different traffic scenarios.

\subsection{Traffic Scenarios and simulation approach}

A simulation model was implemented in OPNET modeller in order to evaluate the performance of multi-class, multi-rate services. Four different service classes were identified. Each service class is characterised by a number of up-link slots that are requested by the MAC layer and an ATM rate which is used for the ATM layer resource utilisation calculations. The main objective of this work is the evaluation of the resources that are available to be used by the ABR service. Therefore, all ABR services that are accepted at the MAC layer have no resource limitation at the ATM layer (i.e. their ATM rate is always zero) and there is no new call blocking at the ATM layer for any service. However, calls that cannot be admitted at MAC layer are blocked and cleared from the system. The

\begin{tabular}{|c|c|c|c|c|c|}
\hline Service Class & MAC Slots & ATM parameters & ATM Service Class & $\begin{array}{c}\text { Traffic } \\
\text { Scenario }\end{array}$ & $\begin{array}{c}\text { Mean Call Arrival } \\
\text { Rate }\left(\lambda_{i}\right)\end{array}$ \\
\hline Class 0 & 1 & $\begin{array}{l}\mathrm{PCR}=1, \text { activity } \\
\text { factor }=0.4\end{array}$ & $\begin{array}{l}\text { CBR, Voice, CLR=10E-3, } \\
\text { 30ms max queuing delay }\end{array}$ & I \& II & Variable \\
\hline Class 1 & $\begin{array}{l}2 \\
4\end{array}$ & $\begin{array}{l}\mathrm{MCR}=2 \\
\mathrm{MCR}=4\end{array}$ & $\begin{array}{l}\text { ABR } \\
\text { ABR }\end{array}$ & $\begin{array}{l}\text { I } \\
\text { II }\end{array}$ & $\begin{array}{l}\text { Variable } \\
\text { Variable }\end{array}$ \\
\hline Class 2 & 4 & $\mathrm{SCR}=4$ & nrt-VBR, Data & I \& II & Variable \\
\hline Class 3 & 10 & $\begin{array}{c}\mathrm{PCR}=10, \mathrm{SCR}=4, \\
\mathrm{MBS}=300 \text { cells }\end{array}$ & $\begin{array}{c}\text { rt-VBR, Video, CLR=10E- } \\
5,30 \mathrm{~ms} \text { max queuing delay } \\
\text { at each layer (MAC and } \\
\text { ATM) }\end{array}$ & I \& II & Variable \\
\hline
\end{tabular}

Table 1: Simulation service scenarios, definition of classes and parameters.

system capacity, for the simulation scenarios presented in Table 1 , was set to $\mathrm{C}=100$ up-link slots.

Two different traffic scenarios were simulated. In traffic scenario I all service classes $(0,1,2,3)$ contribute equally to the total offered load. In traffic scenario II, the contribution of each service class is $20 \%, 40 \%, 20 \%$ and $20 \%$ of the total offered load. New calls are generated according to a Poisson distribution; the call duration follows negative exponential distribution with average value 1 (all the other parameters are normalised to this value).

\section{SiMULATION RESULTS AND DISCUSSION}

The call blocking probability as a function of the offered load is presented in Figure 4 and 7 for traffic scenarios I and II respectively. As expected, the service classes that request for a higher number of MAC slots experience higher call blocking rates. The simulation results are in full agreement with those obtained by analysis (see equations 1 and 2) therefore, the simulation model was used to record very useful statistics that cannot be easily provided by analysis. As shown in Figure 7, the service classes 1 and 2 (traffic scenario II) experience the same call blocking rate although they represent different ATM service classes (ABR and nrt-VBR). Therefore, if there is noadmission control applied by the network, the traffic from different service classes is regulated only by the MAC layer. However, from the ATM resource utilisation statistics that are plotted in Figure 5 and Figure 8 for both traffic scenarios, it is clearly shown that different admission control policies are applicable at the ATM layer. 


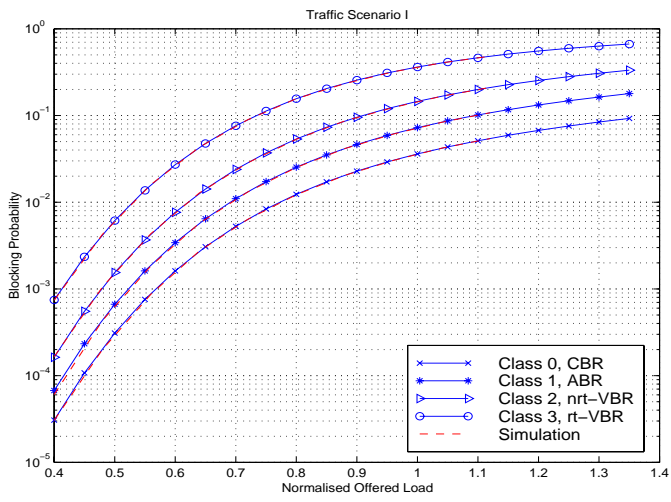

Figure 4: Call blocking rates, scenario I

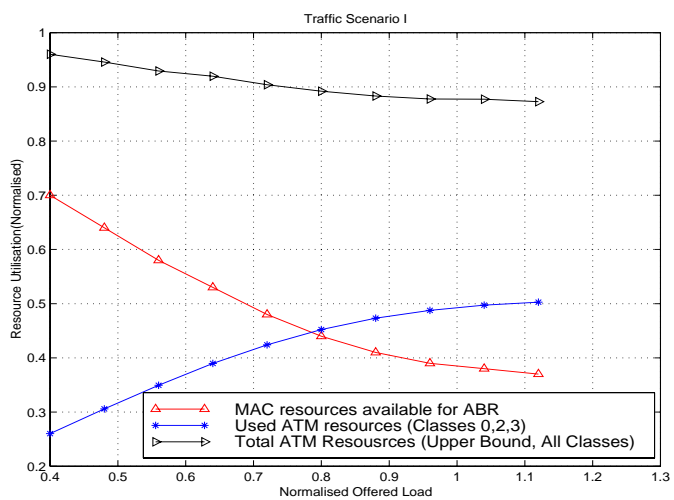

Figure 6: ABR Res. Availability, scenario I

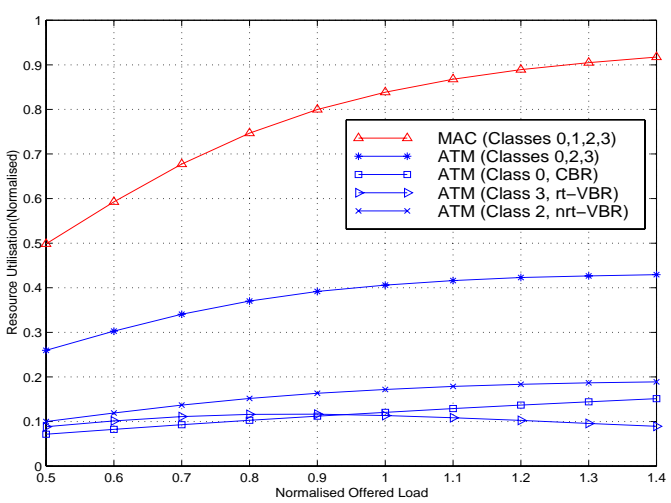

Figure 8: Resource Utilisation, scenario II

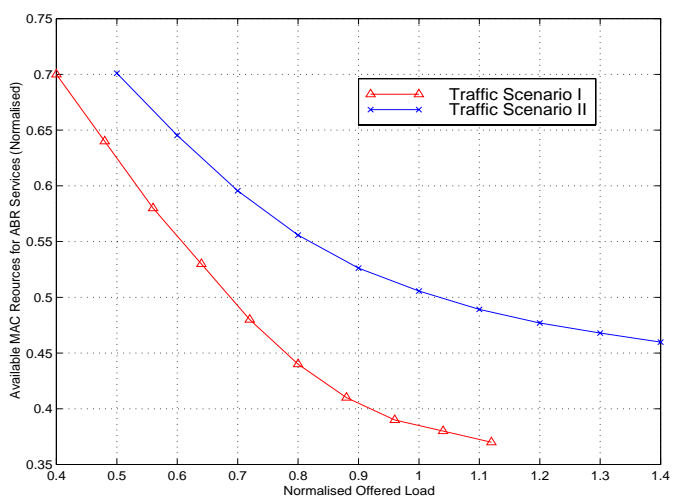

Figure 10: ABR Res. Availability at MAC layer

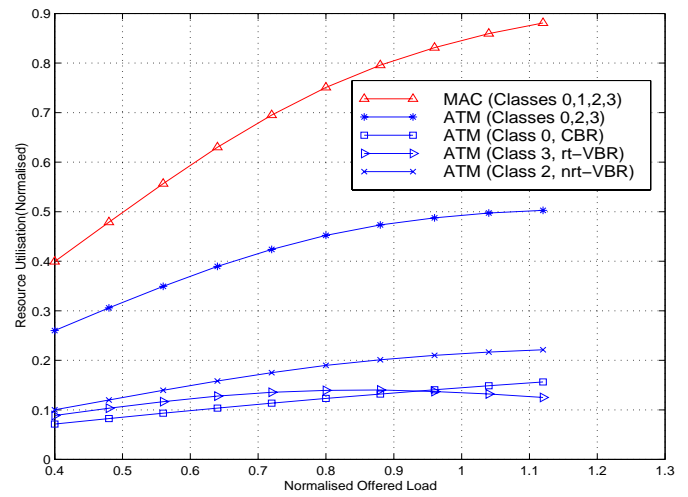

Figure 5: Resource Utilisation, scenario I

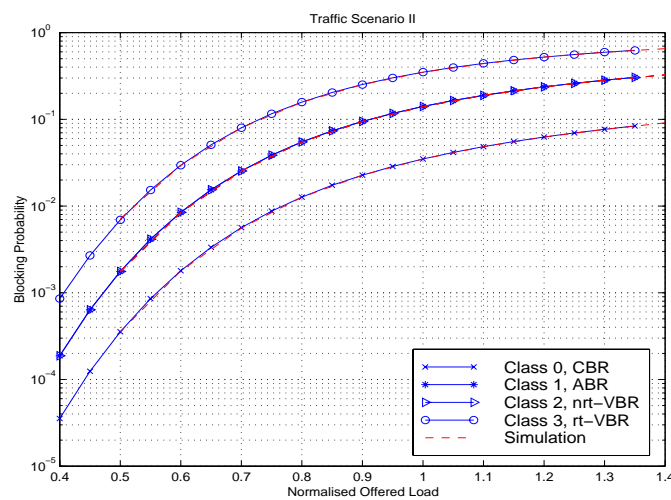

Figure 7: Call blocking rates, scenario II

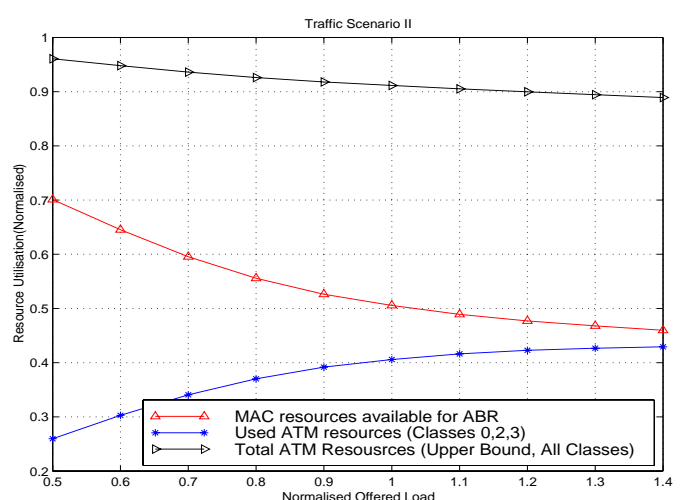

Figure 9: : ABR Res. Availability, scenario II

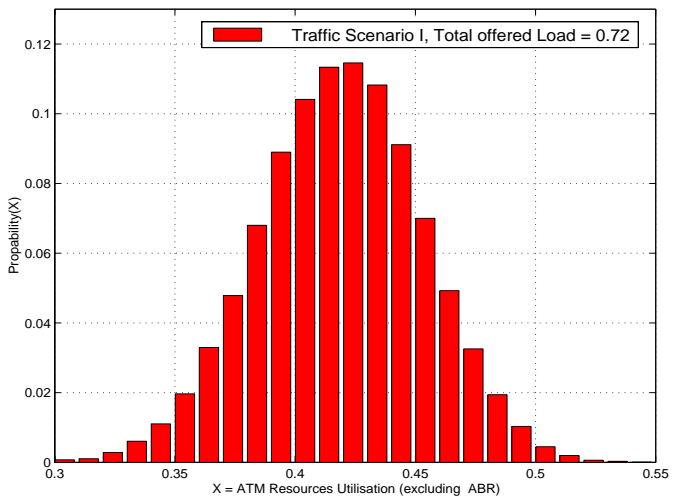

Figure 11: ATM resource utilisation, classes $(0,2,3)$ 
Since the function that calculates the resource utilisation for the ABR service at the ATM layer was unknown, the resource utilisation statistics from all the service classes excluding the ABR were collected and presented in Figure 6 and Figure 9 for traffic scenarios I and II respectively. By adding these graphs to the graphs that represent the resources that are available at the MAC layer for the ABR services (see Figure 10) the upper bounds of the total ATM resource utilisation are clearly shown. These values are normalised to the overall MAC resources. The Probability Density Function (PDF) of the ATM resource utilisation excluding the ABR services is shown in Figure 11 (traffic scenario I, total offered load $=0.72$ ). From these results, we can easily plot the PDFs of the ABR resource availability at the ATM layer for different values of the overall ATM resources. In fact, any connection admission control algorithm that is applied to the ABR service will try to restrict the switch capacity requirements by taking into account the maximum multiplexing gain that can be achieved. In any case, the overall switch capacity should be less than the upper bound values shown in Figure 6 and Figure 9 for the traffic scenarios I \& II.

\section{SUMMARY}

In this paper, an overview of the network aspects of the future Satellite-ATM networks is given, concentrating on the resource management and the traffic control issues. A block diagram of the control functions that are distributed between the space and the ground segment is presented along with the mapping of the ATM layer services to the MAC layer service classes. In addition, a new methodology for evaluating the performance of multi-rate, multi-class services is described. It calculates the call blocking probability per service class at the MAC layer and the ATM resources utilisation. Assuming a MAC layer that supports bandwidth on demand, all the available ATM resources can be allocated to the ABR service without affecting the GoS of the rest of the service classes. Simulation results are presented for two traffic scenarios. Future work includes the addition of a traffic model for the nrt-VBR service in order to include the additional multiplexing gain at the ATM layer.

\section{REFERENCES}

[1] http://www.infowin.org/ACTS/

[2] http://www.ee.surrey.ac.uk/CCSR/ACTS/

[3] E.Elizondo, F.Gargione, R.Gobbi and K.Shockey, "ASTROLINK system overview", Second Kaband Utilization Conference and International Workshop on SCGI, Florence, Italy, 24,26 September 1996.

[4] R. G. Leamon et all, "CYBERSTAR", $3^{\text {rd }}$ Ka-band Utilisation Conference, Sorrento, Italy, 15-18 September 1997.

[5] http://www.teledesic.com

[6] T.Otsu K.Okada Y.Fukuyo M. Minomo, "Ka-Band Satellite Communication Systems Operating through NTT's Communication Satellite N-STAR", $3{ }^{\text {rd }}$ Ka-band Utilisation Conference, Sorrento, Italy, 15-18 September 1997.

[7] B. Le Stradic, M.Vaissiere, N. Boudier, O. Bowles, "The WEST Project: Exploiting the Ka-band Spectrum to Develop the Global Information Infrastructure", ${ }^{\text {rd }}$ Ka-band Utilisation Conference, Sorrento, Italy, 15-18 September 1997.

[8] G. Losquadro, "The EUROSKYWAY System for Interactive Multimedia Operating with FeedBack Aided Traffic Management", $17^{\text {th }}$ AIAA International Communications Satellite Systems Conference and Exhibit, Yokohama, Japan, 23-27 February 1998.

[9] I. Mertzanis., R. Tafazolli, B.G. Evans: Protocol Architecture Scenarios for Satellite and B-ISDN Network Integration, $3^{\text {rd }}$ Ka Band Utilisation Conference, Sorrento, Italy, September 1997 
[10] O. Enomoto, and H. Miyamoto "An analysis of mixtures of multiple bandwidth traffic on time division in switching networks", $7^{\text {th }}$ International Teletraffic Congress Proceedings, pages 635.1$8,1973$.

[11] J. M. Aein "A multi-user-class, blocked-calls-cleared demand access model”, IEEE Transactions on Communications 26(3): 378-385,1978.

[12] J. W. Roberts, "A service system with heterogeneous user requirements - application to multiservice telecommunication systems", G. Pujolle Performance of Data Communication Systems and their Applications, pages 423-431, North Holland-Elsevier Science Publishers, 1981.

[13] af-tm-0056.000,ATM Forum, “Traffic Management Specification Version 4.0”, April 1996.

[14] D. Anick, D. Mitra, M. M. Sondhi, Stohastic theory of a data handling system with multiple sources, Bell Systems Technical Journal, vol.61, no.8, pp.10-18, 1974.

[15] B. Maglaris, D. Anastassiou, P. Sen, G. Karlsson, J. D. Robbins, performance models of statistical multiplexing in packet video communications, IEEE transactions on communications, vol.36, no.7, July1986. 